PROCEEDINGS OF THE AMERICAN MATHEMATICAL SOCIETY

Volume 124, Number 11, November 1996

\title{
ON THE BOUNDARY RIGIDITY PHENOMENON FOR AUTOMORPHISMS OF DOMAINS IN $\mathbb{C}^{n}$
}

\author{
BERNARD COUPET AND ALEXANDER SUKHOV
}

(Communicated by Eric Bedford)

\begin{abstract}
We prove that a piecewise smoothly bounded strictly pseudoconvex domain with a non-compact automorphism group is biholomorphic to the ball. A boundary version of the Schwarz lemma for automorphisms of such a domain is settled.
\end{abstract}

\section{INTRODUCTION}

Let $\Omega$ be a domain in $\mathbb{C}^{n}$ and $p$ a boundary point of $\Omega$. We say that the boundary $b \Omega$ is strictly pseudoconvex piecewise $C^{k}$ smooth and generic at $p$, if there exists a neighborhood $U$ of $p$ in $\mathbb{C}^{n}$ such that

$$
\Omega \cap U=\left\{z \in U \mid r_{j}(z)<0, j=1, \ldots, m\right\},
$$

where every function $r_{j}(z)$ is $C^{k}$ smooth and strictly plurisubharmonic on $U$, $r_{j}(p)=0, j=1, \ldots, m$, and $\bar{\partial} r_{1} \wedge \ldots \wedge \bar{\partial} r_{m} \neq 0$ on $U$. Thus, $\Omega \cap U$ is the wedge with generic edge

$$
M=\left\{z \in U \mid r_{j}(z)=0, \quad j=1, \ldots, m\right\}
$$

of codimension $m$. A bounded domain $\Omega \subset \subset \mathbb{C}^{n}$ is said to be a strictly pseudoconvex domain with piecewise $C^{k}$ smooth generic boundary if $b \Omega$ is a topological manifold and there exists a finite covering of $b \Omega$ by open neighborhoods $U_{i}$ such that every $\Omega \cap U_{i}$ has the form (1) (with $r_{j}(z)$ and $m$ depending on $i$ ). In what follows $\operatorname{Aut}(\Omega)$ denote the holomorphic automorphism group of $\Omega$ with the standard compact open topology.

Our first result is the following

Theorem 0.1. Let $\Omega \subset \subset \mathbb{C}^{n}$ be a strictly pseudoconvex domain with piecewise $C^{2}$ smooth generic boundary. Assume that $\operatorname{Aut}(\Omega)$ is non-compact. Then $\Omega$ is biholomorphic to the unit ball $\mathbb{B}$ of $\mathbb{C}^{n}$.

Together with well-known results of Pinchuk [24] this immediately implies

Theorem 0.2. Let $\Omega \subset \subset \mathbb{C}^{n}$ be a strictly pseudoconvex domain with piecewise $C^{2}$ smooth, but non-smooth, generic boundary. Then $\operatorname{Aut}(\Omega)$ is compact.

Received by the editors May 1, 1995.

1991 Mathematics Subject Classification. Primary 32E35, 32A40.

(C)1996 American Mathematical Society 
The above theorems will work together with the following boundary rigidity principle.

Theorem 0.3. Let $\Omega \subset \subset \mathbb{C}^{n}$ be a strictly pseudoconvex domain with piecewise $C^{k}$ $(k>3)$ smooth generic boundary and let $\operatorname{Aut}(\Omega)$ be compact. Suppose that $p \in b \Omega$ is a boundary point, and $f \in \operatorname{Aut}(\Omega)$ is an automorphism such that

$$
f(z)=z+o(|z-p|) \text { as } z \rightarrow p .
$$

Then $f(z) \equiv z$.

From Theorems 0.1, 0.2, 0.3 we will get our main result.

Theorem 0.4. Let $\Omega \subset \subset \mathbb{C}^{n}$ be a bounded strictly pseudoconvex domain with piecewise $C^{k}(k>3)$ smooth generic boundary. Assume that $p \in b \Omega$ is a boundary point, $f \in \operatorname{Aut}(\Omega)$ is an automorphism and

$$
f(z)=z+o\left(|z-p|^{2}\right) \text { as } z \rightarrow p .
$$

Then $f(z) \equiv z$.

Theorem 0.1 generalizes the well-known results on characterization of smoothly bounded domains with non-compact automorphism group [28], [30]. Our proof is based on Frankel's blow up technique [13]. This approach has been intensively exploited by Kim [18], [19]. It would be of interest to adapt the scaling method of Pinchuk [25] in order to get the purely local version of Theorem 0.1 ; now we are ready to do it for the case where all edges (2) in $b \Omega$ have codimension $\leq 2$ (the details are rather complicated and we do not include them in this paper). We note that Kim [20] investigated the asymptotic behavior of the sectional curvature of the Bergman metric of a piecewise smoothly bounded domain using the arguments close to Pinchuk's scaling (we refer the reader to [14] for the profound discussion of the "blow-up philosophy").

Theorem 0.4 can be treated like a boundary version of the Schwarz lemma. For the case where $p$ is an interior point of $\Omega$ it relies on the classical Cartan theorem [22]. The boundary version of this rigidity phenomenon was discovered by Krantz [17] and then developped by Burns and Krantz [7] and Huang [16] provided $\Omega$ is a smoothly bounded pseudoconvex domain with standard restrictions (strict pseudoconvexity, condition $R$, etc.). It is worth to remark that in [7] arbitrary holomorphic self-mappings of $\Omega$ were treated also. The methods of these papers rely on deep properties of the Bergman metric and the Lempert theory of extremal disks. In this note we will show that (for the automorphism case) this boundary rigidity phenomenon has a simple nature and can be deduced from the classical Bochner linearization theorem [5].

We express our thanks to the referee whose remarks and suggestions improved the first version of the present article.

\section{Compactness of the Automorphism group}

In this section we shall prove Theorem 0.1. We begin with a geometric remark about strictly pseudoconvex domains with piecewise smooth boundary. This is well known for strictly pseudoconvex domains.

Proposition 1.1. Let $\Omega$ be a strictly pseudoconvex domain with piecewise smooth boundary, and let $p$ be a boundary point of $\Omega$. Then, there exists a local holomorphic 
change of coordinate $\Phi$ and a neighborhood $U$ of $p$ such that $\Phi(p)=0$ and $\Phi(\Omega \cap U)$ is convex.

Proof. By definition, there exist strictly plurisubharmonic functions $r_{j}(1 \leq j \leq m)$ defined on a neighborhood $U$ of $p$ such that $\Omega \cap U$ has the form (1).

We introduce the mapping $\alpha: z \mapsto w$ defined by

$$
\begin{aligned}
& w_{j}=\sum_{j=1}^{n} \frac{\partial r_{j}}{\partial z_{k}}(p)\left(z_{k}-p_{k}\right) \quad \text { for } 1 \leq j \leq m, \\
& w_{j}=z_{j}-p_{j} \quad \text { for } m+1 \leq j \leq n .
\end{aligned}
$$

Since one can assume the matrix $\left(\frac{\partial r_{j}}{\partial \zeta_{k}}(p)\right)_{1 \leq j \leq m, 1 \leq k \leq m}$ to be invertible, $\alpha$ is a change of variables. In these coordinates $p$ is 0 and the Taylor expansions of functions $r_{j}(1 \leq j \leq m)$ are :

$$
r_{j}(w)=2 \operatorname{Re}\left(w_{j}\right)+2 \operatorname{Re} B_{j}(w)+H_{j}(w)+R_{j}(w),
$$

where $B_{j}$ is a bilinear form, $H_{j}$ is a positive definite hermitian form, and $R_{j}$ is $o\left(|w|^{2}\right)$. Now, we introduce the mapping $\beta: w \rightarrow \tilde{w}$ defined by the equations

$$
\begin{aligned}
& \widetilde{w}_{j}=w_{j}+B_{j}(w) \quad \text { for } 1 \leq j \leq m, \\
& \widetilde{w}_{j}=w_{j} \quad \text { for } m+1 \leq j \leq n .
\end{aligned}
$$

As each $B_{j}$ is bilinear, $\beta$ is a change of coordinates near 0 . Moreover, in these coordinates, the Taylor expansions of functions $r_{j}(1 \leq j \leq m)$ are

$$
r_{j}(\widetilde{w})=2 \operatorname{Re}\left(\widetilde{w}_{j}\right)+\widetilde{H}_{j}(\widetilde{w})+\widetilde{R}_{j}\left(|\widetilde{w}|^{2}\right),
$$

where $\widetilde{H}_{j}$ is a hermitian form and $\widetilde{R}_{j}$ is $o\left(|w|^{2}\right)$. Let $\Phi=\beta \circ \alpha$. In the local coordinates given by $\Phi$, each function $r_{j}$ is convex near 0 and thus $\Phi(\Omega \cap U)$ is convex.

For the rest of this section, we consider the situation of Theorem 0.1. By the non-compactness of Aut $(\Omega)$, there exists a sequence $\left(f^{\nu}\right)_{\nu}$ of automorphisms of $\Omega$ and a point $z_{0}$ of $\Omega$ such that $\left(f^{n}\left(z_{0}\right)\right)_{n}$ converges to a boundary point $p$. First of all, we have the following essential property :

Lemma 1.2. The sequence $\left(f^{\nu}\right)_{\nu}$ converges uniformly on compact subsets of $\Omega$ to the constant mapping $z \equiv p$.

Proof. Remark that the boundary of $\Omega$ does not contain any analytic set of positive dimension since the functions $r_{j}$ are strictly plurisubharmonic. Thus, the lemma follows from the maximum principle since $\left(f^{\nu}\right)_{\nu}$ contains some converging sequence by Montel's theorem : any limit point takes the boundary value $p$ at the interior point $z_{0}$.

Now, we describe Frankel's blowing up method [13]. We may use this method because Proposition 1.1 gives that the domain is $h$-convex in Frankel's sense [13]. We may assume that $p$ is the point 0 . Since $\left(f^{\nu}\right)_{\nu}$ uniformly converges to zero on compact subsets of $\Omega$, the domains $\Omega^{\nu}=\left(f^{\nu}\right)^{-1}(\Omega \cap U)$ contain any fixed compactum of $\Omega$ for $\nu$ sufficiently large and $\Omega=\bigcup_{\nu} \Omega^{\nu}$. The mappings $\tilde{f}^{\nu}=\Phi \circ f^{\nu}$ from $\Omega^{\nu}$ onto $\Phi(\Omega \cap U)$ are biholomorphic, and the sequence $\tilde{f}^{\nu}$ converges uniformly on each compact subset of $\Omega$ to zero. In what follows, we shall omit the tilde. 
Set $p^{\nu}=f^{\nu}\left(z^{0}\right)$ and introduce the mappings $A^{\nu}$ and $g^{\nu}$ defined by $A^{\nu}(z)=$ $\left[d f^{\nu}\left(z_{0}\right)\right]^{-1}\left(z-p^{\nu}\right)$ and $g^{\nu}=A^{\nu} \circ f^{\nu}$. Frankel's results [13] allow us to prove the following :

Lemma 1.3. There exists some subsequence of the sequence of convex domains $D^{\nu}=A^{\nu}[\Phi(\Omega \cap U) \cap \varepsilon \mathbb{B}]$ converging to a convex domain $D$ (in the local Hausdorff sense) for any small $\varepsilon>0$. Moreover, after passing to a subsequence if necessary, the sequence $\left(g^{\nu}\right)$ converges to a biholomorphism from $\Omega$ onto $D$.

Proof. It is important to note that $g^{\nu}\left(z_{0}\right)=0$ is a fixed point. Frankel [13] proved that the sequence $\left(g^{\nu}\right)$ is normal on $\Omega$ and, passing to a subsequence if necessary, converges to a biholomorphism $g$ of $\Omega$ to a convex domain $g(\Omega)$. Moreover there exists some subsequence of convex domains $D^{\nu}=A^{\nu}[\Phi(\Omega \cap U) \cap \varepsilon \mathbb{B}]$ converging to a convex domain $D$ (in the local Hausdorff sense) for any small $\varepsilon$. In fact, we have $g(\Omega)=D$. In Frankel's paper, this is completely proved only for convex domains $\Omega$. But this is true also in our situation. Indeed, thanks to the convexity of the converging sets $D^{\nu}=A^{\nu}[\Phi(\Omega \cap U) \cap \varepsilon \mathbb{B}]$, any compact subset of $D$ is contained in $D^{\nu}$ for $\nu$ sufficiently large. Then, if we can consider the inverse mapping $h^{\nu}=\left(g^{\nu}\right)^{-1}$, this sequence $\left(h^{\nu}\right)$ is well defined on any compact subset of the domain $D$ and, as $\Omega$ is completely hyperbolic, is also normal ; we may assume its uniform convergence on compact subsets of $D$ to a holomorphic mapping $h$. Since $h^{\nu}(0)=z_{0}$ belongs to $\Omega$ for any $\nu$, and the boundary of $\Omega$ does not contain any analytic set of positive dimension, $h$ is a map from $D$ into $\Omega$. The relation $g^{\nu} \circ h^{\nu}=I d$ for any index $\nu$ gives easily $g(\Omega)=D$. The lemma is proved.

The difficulty to exploit the former result is that $D$ is unknown. We are going to prove that, in our situation, $D$ is an unbounded realization of the ball and necessarily $\Omega$ is smooth.

Set $R^{\nu}=d f^{\nu}\left(z_{0}\right)$. Since the sequence $\left(R^{\nu}\right)$ goes to zero, the sequence of domains $\left(R^{\nu}\right)^{-1}(\varepsilon \mathbb{B})$ goes to $\mathbb{C}^{n}$. Thus, in order to identify the domain $D$, it suffices to follow the behavior of the defining functions $r_{j}(1 \leq j \leq m)$ under the blowing up $A^{\nu}$.

For $\nu$ sufficiently large, $A^{\nu}[\Phi(\Omega \cap U) \cap \varepsilon \mathbb{B}]$ is defined by

$$
\left\{w \in\left(R^{\nu}\right)^{-1}(\varepsilon \mathbb{B}) \mid r_{j}^{\nu}(w)=r_{j}\left(p^{\nu}+R^{\nu} w\right)<0,1 \leq j \leq m\right\}
$$

with $p^{\nu}=f^{\nu}\left(z_{0}\right)$.

Using estimates for the Kobayashi infinitesimal metric [27]: $K_{\Omega}(z, v) \geq$ $C_{1}|v| \operatorname{dist}(z, b \Omega)^{-1 / 2}$ and the invariance property under biholomorphic mappings, we get: $\left\|R^{\nu}\right\| \leq C\left(\delta^{\nu}\right)^{1 / 2}$, where $\delta^{\nu}$ is the distance of $p^{\nu}$ to the boundary of $\Omega$, and $C$ a constant independent of $\nu$. We may assume that the sequence $\left(R^{\nu} /\left(\delta^{\nu}\right)^{1 / 2}\right)$ converges.

By Taylor's formula, we have (for each $1 \leq j \leq m$ )

$$
r_{j}^{\nu}(w)=q_{j}^{\nu}+\operatorname{Re}\left\langle\partial r_{j}\left(p^{\nu}\right), R^{\nu}(w)\right\rangle+H_{j}^{\nu}\left(\ell_{1}^{\nu}(w), \ldots, \ell_{n}^{\nu}(w)\right)+o\left(\delta^{\nu}\right)
$$

uniformly on any compactum, where $q_{j}^{\nu}=r_{j}\left(p^{\nu}\right), \ell_{1}^{\nu}, \ldots, \ell_{n}^{\nu}$ are the components of $R^{\nu}$ and $H_{j}^{\nu}$ is a positive defined hermitian form.

It is convenient to make the linear change of variables defined by

$$
\begin{aligned}
& \tilde{w}_{j}=\sum_{j=1}^{n} \frac{\partial r_{j}}{\partial z_{k}}\left(p^{\nu}\right) w_{k} \quad \text { for } 1 \leq j \leq m, \\
& \tilde{w}_{j}=w_{j} \quad \text { for } m+1 \leq j \leq n .
\end{aligned}
$$


When $\nu$ goes to infinity, this linear mapping tends to the identity and so, it is invertible. In this coordinates, we have

$$
r_{j}^{\nu}(w)=q_{j}^{\nu}+2 \operatorname{Re} \tilde{\ell}_{j}^{\nu}(w)+\widetilde{H}_{j}^{\nu}\left(\tilde{\ell}_{1}^{\nu}(w), \ldots, \tilde{\ell}_{n}^{\nu}(w)\right)+o\left(\delta^{\nu}\right),
$$

where $\widetilde{H}_{j}^{\nu}$ is a positive definite hermitian form. Moreover the sequence $\left(\tilde{\ell}_{j}^{\nu} /\left(\delta^{\nu}\right)^{1 / 2}\right)_{\nu}$ converges for each $j$; let $\tilde{\ell}_{j}$ be the limit.

As the functions $r_{j}$ are smooth, there exists a constant $C$ such that for any $w$ in $\Omega, \operatorname{dist}(w, b \Omega) \leq C \inf \left|r_{j}(w)\right|$. Thus, the sequence $\left(\delta^{\nu} /\left|q_{j}^{\nu}\right|\right)_{\nu}$ is bounded and we also may assume that it converges to a positive number denoted by $\delta_{j}$. It follows that the quantity $q_{j}^{\nu}+\widetilde{H}_{j}^{\nu}\left(\tilde{\ell}_{1}^{\nu}(w), \ldots, \tilde{\ell}_{n}^{\nu}(w)\right)+o\left(\delta^{\nu}\right)$ is $O\left(\left|q_{j}^{\nu}\right|\right)$ on compact sets.

Since 0 is an interior point of $D$, by the convexity of $r_{j}^{\nu}$ there exists a ball $B \subset D$ centered at the origin such that $r_{j}^{\nu}<0$ on $B$ for $\nu$ large, and then the real part of linear forms $\lambda_{j}^{\nu}$ defined by $\lambda_{j}^{\nu}(w)=\widetilde{\ell_{j}^{\nu}}(w) / q_{j}^{\nu}$ is bounded from above on this ball. Thus, we may assume also that this sequence of linear forms converges. Let $\lambda_{j}$ be the limit.

It follows that the functions $\widetilde{r}_{j}^{\nu}=r_{j}^{\nu} / q_{j}^{\nu}$ converge uniformly on compact sets of $\mathbb{C}^{n}$ to a function $\rho_{j}$ defined by $\rho_{j}(w)=-1+2 \operatorname{Re} \lambda_{j}(w)+\delta_{j} H_{j}\left(\tilde{\ell}_{1}(w), \ldots, \tilde{\ell}_{n}(w)\right)$, where $H_{j}$ is the hermitian part of the Taylor expansion of the function $r_{j}$ at 0 . Then $D$ is the set $\left\{w \in \mathbb{C}^{n} \mid \rho_{j}(w)<0,1 \leq j \leq m\right\}$.

Now, we have to determine some relations between the linear forms $\lambda_{1}, \ldots, \lambda_{m}$. We consider the indices such that $\delta_{j}$ is 0 and we way assume that there exists an integer $s$ such that $\delta_{j}$ is not 0 if $j \leq s$, and $\delta_{j}$ is 0 if $s+1 \leq j \leq m$. We claim that $\tilde{\ell}_{j}$ is 0 for $1 \leq j \leq s$. Indeed, as $\frac{\partial r_{j}}{\partial z_{k}}(0)=\delta_{j k}$ and the sequence $\left(R^{\nu} /\left(\delta^{\nu}\right)^{1 / 2}\right)$ converges, the sequence $\left(\lambda_{j}^{\nu} q_{j}^{\nu} /\left(\delta^{\nu}\right)^{1 / 2}\right)$ converges to $\tilde{\ell}_{j}$. For $1 \leq j \leq s,\left|q_{j}^{\nu}\right|$ is equivalent to $\frac{\delta^{\nu}}{\delta_{j}}$ and $\lambda_{j}^{\nu} q_{j}^{\nu} /\left(\delta^{\nu}\right)^{1 / 2}$ to $\frac{\left(\delta^{\nu}\right)^{1 / 2}}{\delta_{j}} \lambda_{j}^{\nu}$ which converges to 0 . Thus $\tilde{\ell}_{j}$ is 0.

For $s+1 \leq j \leq m$, the two forms $\lambda_{j}$ and $\tilde{\ell}_{j}$ are linearly dependent since it is true for any $\nu$.

Thus, we may assume that there exists an integer $t(s \leq t \leq m-1)$ such that $\lambda_{j}$ is not 0 for $s \leq j \leq t$, and $\lambda_{j}$ is 0 for $t+1 \leq j \leq m$.

Remark that the functions $\rho_{j}$ have the following expression :

$$
\begin{aligned}
\rho_{j}(w) & =-1+2 \operatorname{Re} \lambda_{j}(w)+\delta_{j} H_{j}\left(\lambda_{s+1}(w), \ldots, \lambda_{t}(w), \tilde{\ell}_{t+1}(w), \ldots, \tilde{\ell}_{n}(w)\right) \\
\rho_{j}=-1 \quad \text { for } t+1 \leq j \leq m . & \text { for } 1 \leq j \leq t,
\end{aligned}
$$

We claim that the forms $\lambda_{1}, \ldots, \lambda_{t}, \tilde{\ell}_{t+1}, \ldots, \tilde{\ell}_{n}$ are linearly independent. Indeed, the intersection $E$ of the kernels of these $n$ linear forms is contained inside $D$. As $D$ is hyperbolic, the only complex vector contained in $D$ is $\{0\}$. Thus $E$ is $\{0\}$ and these forms are independent.

By an obvious global change of coordinates, we may assume that $D$ is the set $\left\{w \mid \rho_{j}(w)<0,1 \leq j \leq t\right\}$ with

$$
\rho_{j}(w)=-1+2 \operatorname{Re}\left(w_{j}\right)+\delta_{j} H_{j}\left(w_{s+1}, \ldots, w_{t}, w_{t+1}, \ldots, w_{n}\right)
$$

(remark that the functions $\rho_{j}$ for $j$ greater than $s+1$ are linear).

Now, we study the behavior near the boundary of the biholomorphism $f$ from $D$ onto $\Omega$. 
A point $q$ in the boundary of $D$ is said to be regular if the boundary of $D$ is smooth near $q$, that is to say that there exist a neighborhood $V$ of $q$ and an integer $k$ such that $D \cap V=\left\{w \in V \mid \rho_{k}(w)<0\right.$ with $\left.d \rho_{k}(q) \neq 0\right\}$. We have:

Lemma 1.4. Any biholomorphism from $D$ onto $\Omega$ extends continuously to a neighborhood of any regular point.

Proof. We have the estimate for the Kobayashi infinitesimal metric [27] :

$$
K_{\Omega}(z, v) \geq C_{1}|v| \operatorname{dist}(z, b \Omega)^{-1 / 2}
$$

for any $z$ in $\Omega$ and any $v$ in $\mathbb{C}^{n}$.

Applying the Hopf lemma for piecewise smoothly bounded domain [4] to the function $\rho_{k} \circ f^{-1}$ we get :

$$
\operatorname{dist}(f(w), b \Omega) \leq C \operatorname{dist}(w, b D)^{\beta}
$$

for some real number $\beta \leq 1$ and $w$ in $D \cap V$. Now, the usual techniques [2], [11], [27] imply that $f$ extends continuously up to the boundary near $q$.

We can complete the proof of Theorem 0.1 by proving $s=t=1$. Suppose that $t>1$. Then, there exists a regular point $q$ in the boundary such that $\rho_{t}(q)=0$. Let $a$ be a point in $b D$ near $q$ and let $\left(a^{\nu}\right)$ be a sequence of points in $D$ converging to $a$. If $v=(1,0, \ldots, 0)$, we have $\rho_{t}\left(a^{\nu}+\zeta v\right)=\rho_{t}\left(a^{\nu}\right)<0$ for any complex number $\zeta$. Since $\rho_{j}(a)<0$ for $1 \leq j \leq t$, there exists $r>0$ such that $a^{\nu}+\zeta v$ belongs to $D$ for $|\zeta|<r$ and any $\nu$.

The estimate (3) applied to the analytic disc $\varphi^{\nu}$ defined by $\varphi^{\nu}: \zeta \rightarrow f\left(a^{\nu}+\zeta v\right)$ gives :

$$
\left|\frac{\partial \varphi^{\nu}}{\partial \zeta}(0)\right|=\left|\frac{\partial f}{\partial z_{1}}\left(a^{\nu}\right)\right| \leq C / r \operatorname{dist}\left(f\left(a^{\nu}\right), b \Omega\right)^{1 / 2} .
$$

As $f$ extends continuously near $a$, we get $\lim _{\nu}\left(\frac{\partial f}{\partial z_{1}}\left(a^{\nu}\right)\right)=0$. That is to say that the holomorphic mapping $\frac{\partial f}{\partial z_{1}}$ extends continuously up to the boundary of $D$. The uniqueness theorem on generic manifold [23] implies that $\frac{\partial f}{\partial z_{1}}$ is 0 on $D$. It is a contradiction since $f$ is biholomorphic. Then $t$ is 1 and $D$ is defined by the function $\rho_{1}=-1+2 \operatorname{Re}\left(w_{1}\right)+H_{1}\left(w_{2}, \ldots, w_{n}\right)$, where $H_{1}$ is positive. As $D$ is hyperbolic, $H_{1}$ is positive definite and so $D$ is biholomorphic to the ball. That proves Theorem 0.1 .

\section{BOUNDARY RIGIDITY}

In this section we will prove Theorem 0.3 ; we suppose that $\Omega$ and $f \in \operatorname{Aut}(\Omega)$ satisfy the hypothesis of Theorem 0.3 .

Let us consider the action of $\operatorname{Aut}(\Omega)$ on $\Omega$. This is a real analytic mapping

$$
\begin{aligned}
& \phi \quad: \quad \Omega \times \operatorname{Aut}(\Omega) \rightarrow \Omega, \\
& \phi \quad: \quad(z, g) \mapsto g(z) .
\end{aligned}
$$

Since every $g \in \operatorname{Aut}(\Omega)$ extends to a homeomorphism of $\bar{\Omega}[27]$, one can also consider $z$ in the boundary and assume that $\operatorname{Aut}(\Omega)$ acts on $\bar{\Omega}$. We obtain the following :

Lemma 2.1. The action $\phi$ extends to a continuous mapping (in both variables $(z, g))$ which maps $\bar{\Omega} \times \operatorname{Aut}(\Omega)$ onto $\bar{\Omega}$. 
For the smooth boundary case similar results were obtained by Greene-Krantz [15], Bell [3], Bedford [2], Barett [1] and the authors [10].

Proof. A simple modification of the proof of Hopf's lemma for piecewise smoothly bounded domains [4] in spirit of [9] shows that there exist $\alpha \geq 1$, a compact set $K$ in $\Omega$ and a constant $C_{0}>0$ such that any negative plurisubharmonic function $\rho$ on $\Omega$ satisfies

$$
|\rho(z)| \geq C_{0}\left(\inf _{K}|\rho(z)|\right) \operatorname{dist}(z, b \Omega)^{\alpha} .
$$

Since $\operatorname{Aut}(\Omega)$ is compact, by Cartan's theorem [22] we have

$$
C_{1}=\inf \left\{r \circ g^{-1}(z) \mid g \in \operatorname{Aut}(\Omega), z \in K\right\}>0,
$$

where $r(z)=\min _{j} r_{j}(z)\left(r_{j}(z)\right.$ being the defining functions (1) of $\left.\Omega\right)$. Now (4) and the standard arguments with Kobayashi metric give that there exists a constant $C_{2}>0$ such that any $g \in \operatorname{Aut}(\Omega)$ satisfies on $\bar{\Omega}$ :

$$
\operatorname{dist}(g(z), g(w)) \leq C_{2} C_{1}^{-1 / 2 \alpha} \operatorname{dist}(z, w)^{1 / 2 \alpha}, \quad \forall z, w \in \Omega .
$$

Therefore, by Ascoli's theorem, any sequence converging in $\operatorname{Aut}(\Omega)$ also converges uniformly on $\bar{\Omega}$. This implies the desired statement.

Let $U$ be a neighborhood of $p$ such that $\Omega \cap U$ has the form (1) and $M$ is defined by (2). We prove :

Lemma 2.2. There exists a neighborhood $W$ of $p$ such that the restriction $f \mid M \cap W$ is a $C^{2}$ diffeomorphism of $M \cap W$.

Proof. We will use the arguments of Forstnerič [12].

For every $q \in U \cap b \Omega$ we denote by $k(q)$ the number of defining functions (1) that vanish at $q$. The function $q \in U \cap b \Omega \mapsto k(q) \in \mathbb{Z}_{+}$is upper semicontinuous. For $s=1, \ldots, m$ we set

$$
M_{s}=\{z \in U \cap b \Omega \mid k(z)=s\} .
$$

Each $M_{s}$ is a locally closed, generic manifold of codimension $s$ in $\mathbb{C}^{n}$. Remark that $M_{1}$ is the set of smooth points in the boundary. Recall that $f$ is a homeomorphism of $\bar{\Omega}$ and $f(p)=p$. We will show that (near $p$ )

$$
f\left(M_{s}\right)=M_{s} \text { for every } s .
$$

We need the following generalization of Pinchuk's results [24] due to Forstnerič [12], Prop. 1.4.

Proposition 2.3. Let $D$ and $D^{\prime}$ be domains in $\mathbb{C}^{n}$ of the form

$$
\left\{\rho_{j}(z)<0, j=1, \ldots, d\right\}
$$

defined by $C^{2}$ p.s.h. functions $\rho_{j}$, resp. $\rho_{j}^{\prime}$, with generic edges $M$, resp. $M^{\prime}$, of class $C^{k}, k>3$. Suppose that the Levi form of every function $\rho_{j}$ is positive definite on the complex tangent bundle $T^{c} M$, and the analogous condition holds for $M^{\prime}$. If $f: D \rightarrow D^{\prime}$ is a biholomorphic mapping that extends continuously to $D \cup M$ and satisfies $f(M) \subset M^{\prime}$, then $M$ and $M^{\prime}$ have the same dimension. 
Now suppose that (5) does not hold. Then there exists a point $\zeta \in M_{s}$ such that $f(\zeta)=\eta \in M_{s^{\prime}}$ for some $s^{\prime} \neq s$. One can assume $s>s^{\prime}$ since the same argument works for $f^{-1}$ at $\eta$. Choose a sufficiently small neighborhood $V \ni \zeta$ in $M_{s}$ and let

$$
s_{1}=\min \{k(w) \mid w=f(z), z \in V\}<s .
$$

Let $\zeta_{1} \in W$ be a point where the minimum occurs. Since $k(w)$ is upper semicontinuous, $k(f(z))=s_{1}$ for any $z$ in a neighborhood $V^{\prime} \subset V$ of $\zeta_{1}$. Hence, $f$ maps the edge $V^{\prime} \subset M_{s}$ into the edge $M_{s_{1}}$ with $s_{1} \neq s$ : a contradiction with Proposition 2.3. Thus, (5) holds.

Since $p \in M=M_{m}, f(M)=M^{\prime}$ is an edge of the largest possible codimension $m$. But $f(p)=p, p \in M^{\prime}$, and, therefore, $M=M^{\prime}$ near $p$. Now, by the results of Forstnerič [12] $f$ extends $C^{2}$ smoothly to $M$ near $p$.

Proof of Theorem 0.3. We denote by $G$ the closure in Aut $(\Omega)$ of the cyclic subgroup $\left(f^{n}\right)_{n \in \mathbb{Z}}$. Then, by Lemmas 2.1 and 2.2 , one can treat $G$ as a compact group of local fixing point diffeomorphisms of $M$ (in the sense of [5]) and the action

$$
\begin{aligned}
& \phi \quad: \quad M \times G \rightarrow M, \\
& \phi \quad: \quad(z, g) \mapsto g(z)
\end{aligned}
$$

is continuous in both variables. Now, the theorem of Montgomery [21] (see also [8]) implies that all partial derivatives of $\phi$ with respect to $z$ are continuous in $(z, g)$ as well. Thus, by the classical Bochner linearization theorem [5], there exists a local smooth change of coordinates on $M$, centered at $p$ and with the identical linear part, such that in the new coordinates the restriction of any automorphism $g \in G$ to $M$ is linear (we emphasize that the Bochner theorem is applied to the action of the diffeomorphism group on $M$, where $p$ is an interior point). Hence, since $d f(p)=\mathrm{id}$, the restriction $f \mid M$ coincides with identity near $p$. It follows by the boundary uniqueness theorem [23] that $f(z) \equiv z$ on $\Omega$. This proves Theorem 0.3 .

\section{Proof of Theorem 0.4}

In view of Theorem 0.3 , it remains to treat the case where $\operatorname{Aut}(\Omega)$ is not compact. It follows from Theorems 0.1 and 0.2 that $\Omega$ is biholomorphic to $\mathbb{B}$ and $b \Omega$ is smooth. By [26] $f$ extends to a $C^{2}$ diffeomorphism of $\bar{\Omega}$. Let $g: \Omega \rightarrow \mathbb{H}=$ $\left\{\operatorname{Im} z_{n}+\left|z_{1}\right|^{2}+\cdots+\left|z_{n-1}\right|^{2}<0\right\}$ be a biholomorphism ; by [26] $g$ is of class $C^{2}(\bar{\Omega})$ and without loss of generality one can assume that $p=0, g(p)=0$. Then $h(z)=g \circ f \circ g^{-1}$ belongs to $\operatorname{Aut}(\mathbb{H})$ and $h(z)=z+o\left(|z|^{2}\right)$ near the origin. Set $z=\left({ }^{\prime} z, z_{n}\right),{ }^{\prime} z=\left(z_{1}, \ldots, z_{n-1}\right)$. It is well known [29] that any fixed origin automorphism of $\mathbb{H}$ has the form

$$
\begin{aligned}
{ }^{\prime} z^{*} & =\lambda U\left({ }^{\prime} z+a z_{n}\right) /\left\{1-2 i\left\langle^{\prime} z, a\right\rangle-(r+i\langle a, a\rangle) z_{n}\right\}, \\
z_{n}^{*} & =\lambda^{2} z_{n} /\left\{1-2 i\left\langle{ }^{\prime} z, a\right\rangle-(r+i\langle a, a\rangle) z_{n}\right\},
\end{aligned}
$$

where $\langle a, b\rangle=\sum_{j=1}^{n-1} a_{j} \bar{b}_{j}, \lambda>0, r \in \mathbb{R}, a \in \mathbb{C}^{n-1}$, and $U$ is a $(n-1) \times(n-1)$ unitary matrix. Since $h(z)=z+o\left(|z|^{2}\right)$, we get immediately that $r=0, a=0, \lambda=1$, $U=\mathrm{id}$, that is to say $h(z) \equiv z$ and then $f=\mathrm{id}$. 


\section{REFERENCES}

[1] D. Barrett, Regularity of the Bergman projection on domains with transverse symmetries, Math. Ann. 258 (1982), 441-446. MR 83i:32032

[2] E. Bedford, Action of the automorphisms of a smooth domain in $\mathbb{C}^{n}$, Proc. AMS 93 (1985), 232-234. MR 86e:32029

[3] S. Bell, Compactness of families of holomorphic mappings up to the boundary, LNM 1268, Springer 1987, pp. 29-42. MR 88k:32066

[4] F. Berteloot, Hölder continuity of proper holomorphic mappings, Studia Mathematica 100 $\mathrm{n}^{\circ} 3$ (1991), 229-235. MR 92i:32030

[5] S. Bochner, Compact groups of differentiable transformations, Ann. Math. 45 (1945), 372381. MR 7:114g

[6] E. Bedford, J.E. Fornaess, Biholomorphic maps of weakly pseudoconvex domains, Duke Math. J. 45 (1978), 711-719. MR 80d:32016

[7] D. M. Burns, S. G. Krantz, Rigidity of holomorphic mappings and a new Schwarz lemma at the boundary, Journal of AMS 7 (1994), 661-676. MR 94j:32016

[8] S. Bochner, D. Montgomery, Groups of differentiable and real or complex analytic transformations, Ann. Math. 46 (1945), 685-694. MR 7:241d

[9] B. Coupet, Uniform extendibility of automorphisms, Contemporary Mathematics AMS (1992), 177-183. MR 93j:32033

[10] B. Coupet, A. Sukhov, Action du groupe des automorphismes, C.R. Acad. Sci. 318 (1994), 117-120. MR 95a:32054

[11] K. Diederich, J.E. Fornaess, Proper holomorphic maps onto pseudoconvex domains with real analytic boundary, Ann. Math. 282 (1988), 681-700. MR 89m:32045

[12] F. Forstneri $\breve{c}$, A reflection principle on strongly pseudoconvex domains with generic corners, Math. Zeit. 213 (1993), 49-64. MR 94b:32042

[13] S. Frankel, Complex geometry of convex domains that cover varieties, Acta Math. 163 (1989), 109-149. MR 90i:32037

[14] S. Frankel, Affine approach to complex geometry, Contemp. Math. 101 (1989), 263-286. MR 91b:32020

[15] R. Greene, S. Krantz, Normal families and the semicontinuity of isometry and automorphism groups, Math. Z. 190 (1985), 455-467. MR 87d:32055

[16] X. Huang, Some applications of Bell's theorem to weakly pseudoconvex domains, Pac. Jour. Math. 158 (1993), 305-316. MR 93m:32032

[17] S. Krantz, Compactness principle in complex analysis, Seminarios I Volumen 3 Uni. Autonoma de Madrid, 1986.

[18] K.T. Kim, Domains with non compact automorphism groups, Contemp. Math. 101 (1989), 249-262. MR 90m:32036

[19] K.T. Kim, Complete localization of domains with non compact automorphism groups, Trans. AMS 319 (1990), 139-153. MR 90i:32035

[20] K.T. Kim, Asymptotic behavior of the curvature of the Bergman metric of the thin domains, Pacif. J. Math. 155 (1992), 99-110. MR 93f:32025

[21] D. Montgomery, Topological groups of differentiable transformations, Ann. Math. 46 (1945), 382-387. MR 7:114h

[22] R. Narasimhan, Several complex variables, Chicago lectures in Mathematics, Univ. Chic. Press, Chicago, 1971. MR 49:7470

[23] S. Pinchuk, Boundary uniqueness theorem of several complex variables, Matem. Zametki 15 $\mathrm{n}^{\circ} 2$ (1974), 205-212.

[24] S. Pinchuk, Holomorphic inequivalence of certain classes of domains in $\mathbb{C}^{n}$, Math. Sb. 111 $\mathrm{n}^{\circ} 1$ (1980), 67-94. MR 81f:32034

[25] S. Pinchuk, The scaling method and holomorphic mappings, Proc. Symp. Pure Math. 52 Part 1 (1991), 151-161. MR 92i:32031

[26] S. Pinchuk, S. Khasanov, Asymptotically holomorphic functions and their applications, Math. USSR Sbornik 62 (1989), 541-550. MR 89f:32009

[27] R. M. Range, On the topological extension to the boundary of biholomorphic maps in $\mathbb{C}^{n}$, Trans. Amer. Math. Soc. 213 (1976), 203-216. MR 52:8504

[28] J. P. Rosay, Sur une caractérisation de la boule parmi les domaines de $\mathbb{C}^{n}$ par son groupe d'automorphismes, Ann. Inst. Fourier 29 (1979), 91-97. MR 81a:32016 
[29] A.G. Vitushkin, Holomorphic mappings and the geometry of hypersurfaces, Encyclop. of Math. Sci. 7 (Several complex variables I), Springer (1990), 160-214. MR 87j:32064; MR 90j:32003

[30] B. Wong, Characterization of the unit ball in $\mathbb{C}^{n}$ by its automorphism group, Invent. Math. 41 (1977), 253-257. MR 58:11521

LATP, CNRS/URA no 225, CMi, Université de Provence, 39, rue F. Joliot-Curie, 13453 Marseille Cedex 13, France 